\title{
Second-Generation Bioceramics in the Treatment of Immature Permanent Teeth: Systematic Review of the Literature
}

\section{Venditti ${ }^{1 *}$, Ragazzoni FM $^{2}$, Venditti A Jr ${ }^{3}$ and Filippo Ragazzoni ${ }^{4}$}

${ }^{1}$ DDS, Specialist in Paediatric Dentistry, Private Practice, Italy

${ }^{2} D D S$, Private Practice, Italy

${ }^{3}$ Dental Hygienist, Private Practice, Italy

${ }^{4}$ Doctor in Medicine and Surgery, Italy

*Corresponding Author: Venditti A, DDS, Specialist in Paediatric Dentistry, Private

Practice, Italy.
Received: June 21, 2021

Published: July 19, 2021

(C) All rights are reserved by Venditti A., et al.

\section{Abstract}

Introduction: The second generation bioceramic materials are inorganic silicates able to stimulate the production of hydroxyapatite. They have antibacterial properties, short grip time, dimensional expansion capacity after hardening, are hydrophilic and have a compression resistance similar to dentine. The aim of the study is a systematic review of the literature on the use of second-generation bioceramics in the treatment of permanent dentary elements at immature apex.

Materials and Methods: A systematic review of the literature has been carried out through the databases Pubmed, Medline, Scopus and Google Scholar according to the guidelines of the PRISMA statement, selecting all published RCT within 10 years until 1 April 2021 regarding the use of second-generation bioceramics in the pulp treatments of immature permanent dental elements.

Results: In the treatment of capping of the vital pulp, 100\% of immature permanent elements treated with second-generation bioceramics maintain pulp vitality and show physiological root development at a follow up of 12 months, compared to $89.36 \%$ of the teeth treated with calcium hydroxide and MTA. In the treatment of apicogenesis, $90.4 \%$ of immature teeth treated with second-generation bioceramics show physiological root development at a follow-up of 24 months, compared to $89.9 \%$ of the elements treated with calcium hydroxide. In the treatment of apecification, the formation of the apical plug to a follow of 24 months takes place in $100 \%$ of cases either using second-generation bioceramics or using MTA. The release of periapical calcium during the curing time is higher using the new bioceramics then MTA. The elements with immature apices, show a greater resistance to the fracture when they are obturated with bioceramics of second generation then MTA. The resistance increases when silicates are used both in the formation of the apical plug and for radicular back filling.

Discussion and Conclusion: Second-generation bioceramics have numerous advantages over conventional materials used in the treatment of immature permanent dental elements, including reduced uptake time, increased dimensional expansion with improved apical seal, greater resistance to compression and fracture and greater biocompatibility with better maintenance of the clean vitality. Keywords: Bioceramic; Immature Teeth; Open Apex; Vital Pulp Cupping; Apexogenesis, Apecification

\section{Introduction}

Bioceramic materials are biocompatible inorganic silicates designed specifically for medical and dental uses [1]. The first medi- cal use occurred in the '60s in the realization of joint prostheses and bone plates. The success of bioceramics in orthopaedics and later in dentistry was due to the discovery of the ability to trigger 
the production of hydroxyapatite. The direct contact of bioceramics with organic phosphate determines the production of silicate gel with the following neoformation of hydroxyapatite [1,2].

\section{Classification}

The classification of bioceramics requires the distinction in materials: bioinert, bioactive and biodegradable. Bioinert materials, such as alumina and zirconium, are unable to interact with the tissues in contact. Bioactive materials, including bioglass, interact with tissues without causing adverse reactions. Degradable bioceramics, such as tricalcium phosphates, are instead reabsorbed and replaced by new materials [3].

\section{Generations of bioceramics}

The first bioceramic used in dentistry was MTA (Mineral-Trioxide-Aggregate), consisting of tricalcium silicate, dicalcium silicate, tricalcium aluminate, calcium sulfate and bismuth oxide [4]. The MTA, defined as first-generation bioceramic, is still used today for the hooding of the pulp, the closure of the perforations, the closure of the beautiful apices and the orthograde and retrograde closure of the mature root canals [5]. The use of MTA, however, has several disadvantages, including: slow curing time, difficult handling, low compressive strength, manifestations of coronal dyschromia due to bismuth oxide and possible toxicity due to heavy metals contained [6]. To address these disadvantages, new second-generation bioceramics were developed in 2007, including Iroot, Endosequence, Total Fill and Total Fill ${ }^{7,8}$. Unlike MTA, second generation bioceramics do not contain calcium aluminate hydrate (responsible for toxicity from heavy metals), do not contain bismuth oxide (responsible for coronal dyschromia) and produce more hydroxyapatite when they come into contact with organic fluids. In 2009, new secondgeneration bioceramic materials were produced, including Biodentine, Bioroot, NeoMta, Grey MTA, Endocem MTA, Endocem ZR and Endoseal $[7,8]$.

\section{Mechanism of action}

Bioceramics are bioactive materials because they have the ability to interact with organic tissues. When they come into contact with endodontic organic fluids, bioactive materials are hydrated and release calcium ions. These ions come into contact with organic phosphates and trigger the formation of hydroxyapatite precursors and then mature hydroxyapatite. During the hardening period, they reach $\mathrm{pH}$ values of around 12.4 resulting in an alkaline etching of the dentinal tubules. The opening of the dentinal tubules allows the neoformed hydroxyapatite to interdigitarsi to the inside, forming the so-called "mineral Infiltration zone" $[9,10]$.

\section{Property}

Bioceramics are bioactive materials, as they are able to induce the formation of hydroxyapatite and the mordanting of the dentinal tubules [10]. They also stimulate fibroblast adhesion when applied in contact with dental pulp. They are biocompatible materials, as they do not induce organic toxicity. Studies show that biocompatibility is greater in second-generation bioceramics than in MTA. This is due to the fact that the composition of the MTA contains heavy metals capable of generating toxicity such as aluminium, which is absent in second-generation materials. The bioceramics have antibacterial properties, thanks to the ph equal to 12 during the period of hardening $[11,12]$. The material with the most antibacterial properties is the Rootsp, able to maintain the antimicrobial action up to 7 days [12]. Second generation bioceramics have the advantage of not causing discoloration of dentary elements, thanks to the absence of bismuth oxide, present in MTA [13]. Another important difference between first and second generation materials is the time taken: the MTA hardens in an average time of 4 hours, while the second generation bioceramics take about 12 minutes [14]. The compression strength is also about $150 \mathrm{Mpa}$ for the MTA and about 300 Mpa for second-generation materials, very similar to natural Dentine [14].

\section{Indications}

Dental bioceramics are distinguished by consistency, composition and use in root repair materials (RRM, Root Repair Materials) and root filling materials (RFM, Root Fill Materials) [15]. The indications of RRM in pediatric dentistry are the therapy of the vital pulp, the closure of the perforations, the seal of the beautiful apices, the root resorptions, and the revascularization pulpare [15]. The indications of the RFM are the use as endodontic sealer and in the technique of cold closing with single cone [15].

Endodontic therapy of the permanent teeth with immature apex

The complete development of the roots takes place about 2 - 3 years after the eruption of the permanent dentary element [16]. When deep carious lesions or trauma associated with pulparous exposures affect permanent dental elements prior to complete root 
formation, endodontic treatments are required to allow for physiological root development [16]. When pulping necrosis of the immature apex tooth element occurs, endodontic therapy should aim at obtaining an apical seal to perform a good three-dimensional root canal filling [16]. The endodontic treatments of the permanent teeth with immature apex are: the direct hooding of the pulp, the apicogenesis, the beekeeping and the revascularization pulpare $[17,18]$. Direct hooding is indicated in cases of iatrogenic or traumatic point exposure, in the absence of spontaneous painful symptoms and bacterial contamination [17]. The most commonly used materials in hooding to stimulate repair phenomena are: calcium hydroxide, MTA, second generation bioceramics and adhesive systems [17]. Apicogenesis is the therapy of choice in the cases of vital dental elements with apices affected by clean exposures of a iatrogenic or traumatic nature, without spontaneous painful symptoms and without signs of periapical radiotransparency. It involves the removal of the chamber pulp, preserving the vitality of the root pulp in order to allow the complete development of the root. The materials used are calcium hydroxide, MTA and bioceramics. In the case of dentary elements with immature apex and necrotic pulparous tissue, the treatments used are pulparetic apecification and revascularization [19]. The apecification consists in the induction of the apical closure through the formation of a barrier of calcific tissue, thanks to the affixing of particular materials, such as calcium hydroxide, MTA and bioceramics [18,19]. Following the formation of the calcium barrier, the root canals are subsequently closed three-dimensionally with gutta-percha and/or endodontic sealers $[18,19]$. The root walls of elements treated with apecification, however, remain thin and little resistant to fracture. In order to overcome this important clinical disadvantage, the technique of revascularization or regeneration pulpare has been developed over the years $[18,19]$. This technique consists in the disinfection and subsequent stimulation of the residual pulparous tissue cells by the formation of a clot in order to induce the continuation of root development leading to thickening of the walls and apical closure $[18,19]$. The materials used in the execution of this technique are MTA and bioceramics $[18,19]$.

\section{Aim of the Study}

The aim of the study is to carry out a systematic review of the Randomized Controlled Trials present in the literature regarding the use of second-generation bioceramic materials in the different endodontic treatment techniques of immature permanent teeth, or the direct capping of the pulp, the apicogenesis, the beekeeping and the clean revascularization.

\section{Materials and Methods}

A systematic review of the literature on the use of second-generation bioceramic materials in the treatment of immature-apex permanent elements has been carried out. The PRISMA (Preferred Reporting Items for Systematic Reviews and Meta-analysis) protocol was followed to compile the systematic review in question [20]. The search engines used for the analysis of bibliographic data were Pubmed, Medline, Google Scolar and Scopus, the search words used were: "(Bioceramic OR Biodentine OR Iroot OR Endosequence OR Totalfill or Bioroot OR NeoMta OR GreyMTA, OR Endoseal OR Endocem) AND (Open apex OR Immature apex OR Immature teeth Or Permanent vital pulp cupping OR Apecification OR Apexogenesis OR Pulp regeneration OR Pulp revascolarization)".

The bibliographic search included data updated to 1 April 2021 without language constraints. The inclusion criteria for the selection of articles were:

1. RCT on the use of second-generation bioceramics in endodontic treatments of immature apex permanent teeth.

2. Studies in vivo and in vitro.

3. Articles published in the last 10 years.

The exclusion criteria were:

1. Endodontic treatments of immature permanent teeth without the use of second-generation bioceramics

2. Clinical trials not randomized, case reports, reviews.

The preliminary bibliographical search, after the insertion of the keywords in the search engines, has allowed the identification of 1141 articles. We proceeded to the elimination of the articles published before the last 10 years, selecting 664 more recent articles. In addition, only RCT articles were included, reducing research to 17 articles. After analyzing all the works in full-text, 11 articles were excluded that did not deal with bioceramics. Six articles suitable for revision were therefore identified, of which one dealt with the hooding of the vital pulp, one with apicogenesis and four with beekeeping. No RCT has been included regarding the use of bioceramics in the treatment of clean revascularization, as they were present in the literature only Clinical Trials without control. 


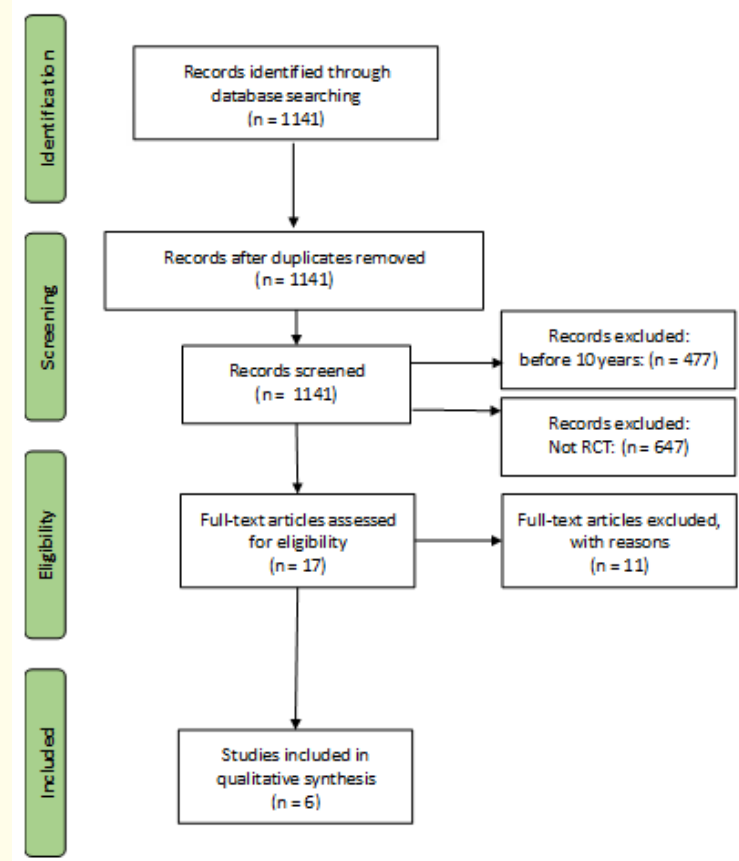

Figure 1: Prisma statement flow-chart.

\section{Results}

The review of the literature has identified 6 RCT articles published in the last 10 years about the use of second-generation bioceramics in the treatment of immature permanent teeth. Of the six articles identified, one article concerned the direct capping of the vital pulp, one article the apicogenesis and four articles the apecification.

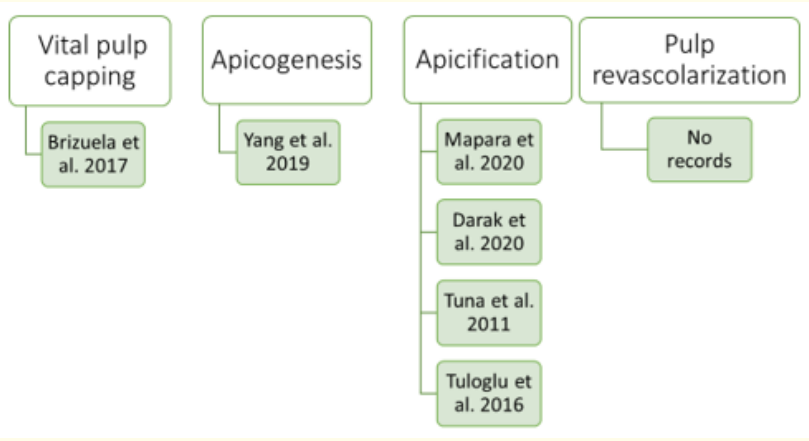

Figure 2: Records identified from review.
Direct pulp capping

Brizuela [21] compared the clinical success of calcium hydroxide, MTA and second generation Bioceramics (Biodentine, Septodont) on a sample of 169 immature apex permanent dental elements with deep caries treated with direct capping of the pulp. The results of the RCT showed for calcium hydroxide success rates of $99.22 \%$ of cases at 3 months, $93.11 \%$ at 6 months and $89.36 \%$ at 12 months. The MTA showed 100\% success in 3-month cases, $91.89 \%$ at 6 months and $86.36 \%$ at 12 months. The biodentine have shown instead the $100 \%$ of success to 3,6 and 12 months (Table 1) [21].

\begin{tabular}{|l|c|c|c|c|}
\hline Success & CH & MTA & Biodentine & Pvalue \\
\hline 3 months & $99,22 \%$ & $100 \%$ & $100 \%$ & 283 \\
\hline 6 months & $93,11 \%$ & $91,89 \%$ & $100 \%$ & 221 \\
\hline 12 months & $89,36 \%$ & $86,36 \%$ & $100 \%$ & 127 \\
\hline
\end{tabular}

Table 1: Brizuela., et al. [21] 2017. Results direct pulp capping.

The results of the study show a statistically significant success difference between calcium hydroxide, first-generation bioceramics (MTA) and second-generation bioceramics (Biodentine), with better results for the last [21].

\section{Apicogenesis}

Yang [22] compared second-generation bioceramics (IRootbp) with calcium hydroxide in apicogenesis treatment in a sample of 110 permanent dental elements with immature apices and crownradicular fracture with pulp exposure. In the 12-month follow-up, the survival of the treated elements, the length of the roots, the thickness of the third coronal and two-thirds apical of the roots and the thickness of the formed dentinal bridge were assessed. The results showed a survival of $89.9 \%$ for the calcium hydroxide group and $90.4 \%$ for the bioceramic group. The radicular length was 14,38 +- 1,43 mm in the calcium hydroxide group and 14,46 +$99 \mathrm{~mm}$ in the bioceramic group. The thickness of the third coronal root was 3,64+- 0,48 $\mathrm{mm}$ in the calcium hydroxide group and 3,76 $+-0,36 \mathrm{~mm}$ in the bioceramic group. The two-thirds apical thickness was 2,84+- 0,42 $\mathrm{mm}$ in the calcium hydroxide group and 2,84 $+-0,84 \mathrm{~mm}$ in the bioceramic group. The tooth bridge thickness was 1,36+- 0,12 $\mathrm{mm}$ in the calcium hydroxide group and 0,97+$0,13 \mathrm{~mm}$ in the bioceramic group (Table 2) [22].

RCT results showed no significant differences between calcium hydroxide and bioceramics in the treatment of apicogenesis, showing high success rates for both materials [22]. 


\begin{tabular}{|l|c|c|c|}
\hline & CH & IRootBP & P-value \\
\hline Success & $89,9 \%$ & $90,4 \%$ & 0.227 \\
\hline Root length & $14,38+-1,43$ & $14,46+-1,99$ & 0.261 \\
\hline $\begin{array}{l}\text { Third coronal root } \\
\text { thickness }\end{array}$ & $3,64+-0,48$ & $3,76+-0,36$ & 0,698 \\
\hline $\begin{array}{l}\text { Apical and medium } \\
\text { root third thickness }\end{array}$ & $2,84+-0,42$ & $2,84+-0,84$ & 0,509 \\
\hline $\begin{array}{l}\text { Thickness of the den- } \\
\text { tinal bridge }\end{array}$ & $1,36+-0,12$ & $0,97+-0,13$ & 0,029 \\
\hline
\end{tabular}

Table 2: Yang., et al [22].

\section{Apicificazione}

Tuloglu [23] compared in vivo MTA and Bioaggregate (secondgeneration bioceramic) in apical plug formation in 26 permanent immature apex incisors with pulparous necrosis associated with trauma. The radiographic parameters analysed were the aspect of the periodontal ligament (LPD), the presence of radicular external resorptions and the possible increase in the size of pre-existing periapical lesions (Table 3).

\begin{tabular}{|l|c|c|}
\hline & MTA & BioAggregate \\
\hline LPD normal & 18 mesi: $100 \%$ & 18 mesi: $100 \%$ \\
\hline $\begin{array}{l}\text { Absence of external } \\
\text { resorptions }\end{array}$ & 18 mesi: $100 \%$ & 24 mesi: $100 \%$ \\
\hline $\begin{array}{l}\text { Increase in periapical } \\
\text { lesion }\end{array}$ & 18 mesi: $0 \%$ & 18 mesi: $100 \%$ \\
& 24 mesi: $0 \%$ & 24 mesi: $0 \%$ \\
\hline
\end{tabular}

Table 3: Tuloglu., et al [23].

The results showed no statistically significant differences between the two materials [23].

Mapara [24] compared in vitro the MTA, Biodentine and Endosequence RRM in the ability to release calcium when used in apical plug formation in beekeeping treatments (Table 4).

The results showed statistically significant differences between materials, showing greater calcium release capacity in second-generation bioceramics and in particular in Endosequence Root Repair Material [24].

\begin{tabular}{|l|c|c|c|c|}
\hline & Days & $\begin{array}{c}\text { Calcium relaise } \\
\mathbf{m g} / \mathbf{L}\end{array}$ & SD & P \\
\hline \multirow{3}{*}{ MTA } & 7 & 5,63 & 0,01 & \\
& 15 & 3,83 & 0,08 & $<0,05$ \\
& 30 & 1,41 & 0,08 & \\
\hline \multirow{3}{*}{ Biodentine } & 7 & 7,02 & 0,04 & \\
& 15 & 4,59 & 0,19 & $<0,05$ \\
\hline \multirow{2}{*}{ Endosequence } & 15 & 1,63 & 0,06 & \\
& 30 & 10,78 & 0,11 & \\
& 30 & 9,54 & 0,06 & $<0.05$ \\
\hline
\end{tabular}

Table 4: Mapara., et al [24].

Tuna [25] evaluated in vitro the fracture resistance of dentary elements with immature apex closed at the apical level and radicular with Bioaggregate, MTA-Angelus, MTA-Proroot and calcium hydroxide.

\begin{tabular}{|l|c|c|c|}
\hline & Mean +- SD & Median & P-value \\
\hline BioAggregate & $37,69+-14,43$ & 30,09 & 0,042 \\
\hline MTA-Angelus & $32,94+-8,15$ & 28,5 & 0,042 \\
\hline MTA - ProRoot & $28,74+-9,49$ & 28,4 & 0,042 \\
\hline Calcium idroxide & $23,18+-8,4$ & 21,6 & 0,042 \\
\hline
\end{tabular}

Table 5: Tuna., et al [25].

The results showed that dental elements treated with Bioaggregate showed increased fracture resistance. Elements treated with calcium hydroxide had the lowest resistance analyzed [25].

Darak [26] compared the fracture resistance of permanent dentary elements with closed immature apex with: 1) MTA in the first apical $5 \mathrm{~mm}$ and subsequent back-filling with MTA; 2) MTA in the first apical $5 \mathrm{~mm}+$ root closure with gutta-percha; 3) Biodentine in the first apical $5 \mathrm{~mm}$ and subsequent back-filling with Biodentine; 4) Biodentine in the first apical $5 \mathrm{~mm}$ and root closure with guttapercha (Table 6). 


\begin{tabular}{|l|c|c|c|}
\hline & Media & DS & P-value \\
\hline MTA apical and back filling & 1034.1 & 65.7 & 0,0 \\
\hline MTA apical + Gutta back filling & 831.6 & 152,3 & 0,0 \\
\hline Biodentine apical and back filling & 1018.4 & 48,6 & 0,0 \\
\hline Biodentine apical + gutta back filling & 806.5 & 119,7 & 0,0 \\
\hline
\end{tabular}

Table 6: Darak., et al [26].

The results show that fracture resistance is greater when the entire immature root canal is filled with MTA or Biodentine than when gutta-percha is used for subsequent root filling 26. Table 7 shows the results of the studies analysed.

\begin{tabular}{|l|c|c|c|c|}
\hline Author & Year & Treatment & Results \\
\hline Brizuela [21] & 2017 & Pulp capping & $\begin{array}{r}\text { Maintenance of pulping vitality and root develop- } \\
\text { ment in 100\% of cases at 12 months. }\end{array}$ & MTA: 89,36\% \\
CH: 89,36\%
\end{tabular}

Table 7: Results.

\section{Discussion}

Second generation bioceramics are biocompatible inorganic silicates designed to cope with the disadvantages of MTA in different endodontic treatments [10-13]. The advantages of the new bioceramic compared to the MTA are: the reduced grip time (12 min vs 4 hours); the best handling of the material; the absence of dental dyschromia due to the absence of bismuth oxide and greater biocompatibility due to the absence of tricalcium aluminate in formulation [10-14]. In endodontic treatment of immature permanent dental elements, second-generation bioceramics show better clinical results than calcium hydroxide and results equal to or better than MTA [21-26]. The use of second-generation bioceramics in the direct capping of the pulp shows better survival results at 6 and 12 months compared to calcium hydroxide and MTA [21]. Treatment of permanent dentures with immature apex and viable pulp by apicogenesis shows overconfident success rates between second generation biodentines and calcium hydroxide [22]. Bioceramic materials have high success rates even in the treatment of apecification of the permanent dentary elements with immature apex and necrotic pulp. Success rates among first- and secondgeneration bioceramics are similar. However, the new bioceramics allow a greater release of calcium during the formation of the apical plug [24]. A common problem of the elements treated with beekeeping is the risk of fracture due to thin and hypodeveloped root walls. Tuna [25] evaluated the fracture resistance of immature permanent tooth elements treated with beekeeping by comparing the use of bioceramics Bioaggregate, MTA-Angel, MTA-Proroot and calcium hydroxide. The results show that dental elements treated with second-generation bioceramics exhibit greater fracture resistance than elements treated with MTA and calcium hydroxide 
[25]. Darak [26] also analyzed the fracture resistance of elements treated with apicogenesis, comparing MTA and Biodentiins both when these materials were used only for the formation of the apical plug that when they were also used for back-root filling. The results showed greater resistance to fracture when MTA or Biodentine was used both at the apical level and for full root filling [26].

\section{Conclusion}

Second-generation bioceramics are today an excellent alternative to conventional materials used in endodontic treatments of permanent teeth elements with immature apex. The results obtained in the analysed Rcts describe important clinical advantages to the use of these materials compared to calcium hydroxide and MTA. However, more Rcts are needed in order to evaluate the clinical results of new bioceramics in the treatment of direct capping of the pulp, apicogenesis, beekeeping and cleaning revascularization.

\section{Bibliography}

1. Marga R and Schwartz R. "Clinical applications of bioceramic materials in endodontics". Endodontic Practice 7.1 (2015): 1-9.

2. Hench L., et al. "Bonding Mechanisms at the Interface of Ceramic Prosthetic Materials". Journal of Biomedical Materials Research 2.1 (1971): 117-141.

3. Niu LN., et al. "A review of the bioactivity of hydraulic calcium silicate cements". Journal of Dentistry 42.5 (2014): 517-533.

4. Torabinejad M., et al. "Physical and Chemical Properties of a New Root- End Filling Material". Journal of Endodontics 21 (1995): 349-353.

5. Ha WN., et al. "Mineral Trioxide Aggregate-A Review of Properties and Testing Methodologies". Materials 10.11 (2017): 1261.

6. Kaur M., et al. "MTA versus Biodentine: Review of Literature with a Comparative Analysis". Journal of Clinical and Diagnostic Research 11.8 (2017): ZG01-ZG05.

7. Gallusi G., et al. "Antibacterial activity of first and latest generation bioceramic sealers on the elimination of enterococcus faecalis: An in vitro study". Journal of Biological Regulators and Homeostatic Agents 34.3-1 (2020): 73-79.

8. Kahler B., et al. "Alkaline Materials and Regenerative Endodontics: A Review". Materials 10.12 (2017): 1389.
9. Niu LN., et al. "A review of the bioactivity of hydraulic calcium silicate cements". Journal of Dentistry 42.5 (2014): 517-533.

10. Atmeh AR., et al. "Dentin-cement interfacial interaction: calcium silicates and polyalkenoates". Journal of Dental Research 91.5 (2012): 454-459.

11. Bósio CC., et al. "Subcutaneous connective tissue reactions to iRoot SP, mineral trioxide aggregate (MTA) Fillapex, DiaRoot BioAggregate and MTA". International Endodontic Journal 47.7 (2014): 667-674.

12. Zhang H., et al. "Antibacterial activity of endodontic sealers by modified direct contact test against Enterococcus faecalis". Journal of Endodontics 35.7 (2009): 1051-1055.

13. Keskin C., et al. "Color stabilities of calcium silicate-based materials in contact with different irrigation solutions". Journal of Endodontics 41.3 (2015): 409-411.

14. Stringhini Junior E., et al. "MTA and biodentine for primary teeth pulpotomy: a systematic review and meta-analysis of clinical trials". Clinical Oral Investigations 23.4 (2019): 19671976.

15. Raghavendra SS., et al. "Bioceramics in endodontics - a review". Journal of Istanbul University Faculty of Dentistry 51.3-1 (2017): S128-S137.

16. Kamel K and Abuzied R. "Managements of immature apex: a review". Mod Research Dentistry 1.1 (2017): 7-27.

17. Guerrero F., et al. "Apexification: A systematic review". Journal of Conservative Dentistry 21.5 (2018): 462-465.

18. Hameed MH., et al. "Management of immature necrotic permanent teeth with regenerative endodontic procedures - a review of literature". Journal of Pakistan Medical Association 69.10 (2019): 1514-1520.

19. Kim SG., et al. "Regenerative endodontics: a comprehensive review". International Endodontic Journal 51.12 (2018): 13671388.

20. Hébert R., et al. "PRISMA-Estrie Group. The effectiveness of the PRISMA integrated service delivery network: preliminary report on methods and baseline data". The International Journal of Integrated Care 8 (2008): e03.

21. Brizuela C., et al. "Direct Pulp Capping with Calcium Hydroxide, Mineral Trioxide Aggregate, and Biodentine in Permanent Young Teeth with Caries: A Randomized Clinical Trial". Journal of Endodontics 43.11 (2017): 1776-1780. 
22. Yang Y., et al. "The effect of partial pulpotomy with iRoot BP Plus in traumatized immature permanent teeth: A randomized prospective controlled trial". Dental Traumatology 36.5 (2020): 518-525.

23. Tuloglu N and Bayrak S. "Comparative evaluation of mineral trioxide aggregate and bioaggregate as apical barrier material in traumatized nonvital, immature teeth: A clinical pilot study". The Nigerian Journal of Clinical Practice 19.1 (2016): 52-57.

24. Mapara PN., et al. "Comparative evaluation of calcium release of the apical plugs formed by mineral trioxide aggregate, Biodentine, and EndoSequence root repair material with and without $2 \%$ triple antibiotic powder: An in vitro study". Journal of the Indian Society of Pedodontics and Preventive Dentistry 38.2 (2020): 132-137.

25. Tuna EB., et al. "Fracture resistance of immature teeth filled with BioAggregate, mineral trioxide aggregate and calcium hydroxide". Dental Traumatology 27.3 (2011): 174-178.

26. Darak P., et al. "Comparative evaluation of fracture resistance of simulated immature teeth and its effect on single visit apexification versus complete obturation using MTA and biodentine". Journal of Family Medicine and Primary Care 9.4 (2020): 2011-2015.

Volume 5 Issue 8 August 2021

(C) All rights are reserved by Venditti A., et al. 